\title{
Finite Temperature Lanczos Method with the Stochastic State Selection and Its Application to Study of the Higgs Mode in the Antiferromagnet at Finite Temperature
}

\author{
Tomo Munehisa \\ Faculty of Engineering, University of Yamanashi, Kofu, Japan \\ Email: munehisa@yamanashi.ac.jp
}

How to cite this paper: Munehisa, T. (2017) Finite Temperature Lanczos Method with the Stochastic State Selection and Its Application to Study of the Higgs Mode in the Antiferromagnet at Finite Temperature. World Journal of Condensed Matter Physics, 7, 11-30.

https://doi.org/10.4236/wjcmp.2017.71002

Received: December 7, 2016

Accepted: February 6, 2017

Published: February 9, 2017

Copyright $\odot 2017$ by author and Scientific Research Publishing Inc. This work is licensed under the Creative Commons Attribution International License (CC BY 4.0).

http://creativecommons.org/licenses/by/4.0/

\begin{abstract}
We propose an improved finite temperature Lanczos method using the stochastic state selection method. In the finite temperature Lanczos method, we generate Lanczos states and calculate the eigenvalues. In addition we have to calculate matrix elements that are the values of an operator between two Lanczos states. In the calculations of the matrix elements we have to keep the set of Lanczos states on the computer memory. Therefore the memory limits the system size in the calculations. Here we propose an application of the stochastic state selection method in order to weaken this limitation. This method is to select some parts of basis states stochastically and to abandon other basis state. Only by the selected basis states we calculate the inner product. After making the statistical average, we can obtain the correct value of the inner product. By the stochastic state selection method we can reduce the number of the basis states for calculations. As a result we can relax the limitation on the computer memory. In order to study the Higgs mode at finite temperature, we calculate the dynamical correlations of the two spin operators in the spin-1/2 Heisenberg antiferromagnet on the square lattice using the improved finite temperature Lanczos method. Our results on the lattices of up to 32 sites show that the Higgs mode exists at low temperature and it disappears gradually when the temperature becomes large. At high temperature we do not find this mode in the dynamical correlations.
\end{abstract}

\section{Keywords}

Higgs Mode, Heisenberg Antiferromagnet, Dynamical Correlation, Finite Temperature Lanczos Method, Stochastic State Selection Method 


\section{Introduction}

The recent discovery of the Higgs particle [1] in the particle physics [2] [3] has stimulated study of the Higgs mode in the condensed matter physics [4]. One can find many experimental reports on the existence of this mode [5]-[11]. Among them we note that the experiment of superconducting films [12] close to the quantum phase transition gives us the strong evidence for the Higgs mode. In addition, theoretical study based on the sigma model, the spin wave theory and other effective models has been active. The purpose of the study is to find experimental conditions to observe the Higgs mode clearly [13]-[22]. Another purpose is to understand the role of this mode near the critical point of the quantum phase transition [23]-[28].

In a previous study [29] we have studied the Higgs mode in the spin-1/2 Heisenberg antiferromagnet on the square lattice at zero temperature. It is well known that many materials realize the Heisenberg antiferromagnet because of its quite simple Hamiltonian. This system has been studied extensively by several numerical methods [30] [31] [32] as well as by the spin wave theory [33]. One motivation of the previous study is to find directly the numerical evidence for the Higgs mode in the quantum antiferromagnet by the reliable method. Another motivation is to investigate how the Higgs mode is induced from the fundamental Hamiltonian. Since the Higgs mode is the resonance state and can couple with two Goldstone-Nambu modes, we have calculated the dynamical correlations of the two spin operators on the finite lattice using the exact diagonalization approach. On the finite lattice we cannot find the resonance itself, but we can find several excited states instead. Taking this into account we have proposed four procedures to find the evidences for the resonance. The results have showed that we can find the Higgs mode in the dynamical correlations of the two spin operators. Also we have clarified differences between the Higgs modes in the $\mathrm{SU}(2)$ symmetry and those in the $\mathrm{U}(1)$ symmetry through the study of the XXZ model [29].

In order to confirm that the Higgs mode exists at finite temperature, we would like to study the Higgs mode in the spin-1/2 Heisenberg antiferromagnet on the square lattice using the finite temperature Lanczos method (FTLM) [34]-[39]. When we apply the FTLM to calculations of the dynamical correlations of the operators at finite temperature, we use the set of the states generated by the Lanczos method to calculate the matrix elements of the operator between these Lanczos states. In the calculation at zero temperature the matrix elements are calculated between the Lanczos states and the ground state. In these calculations we need to keep the ground state, but we do not need to keep the Lanczos states after we calculate the matrix elements. In the calculations at finite temperature, on the other hand, we generate two kinds of sets of the Lanczos states. We then calculate the matrix elements between the Lanczos states of one set and the Lanczos states of another set. For these calculations we have to keep two kinds of sets of the Lanczos states on computer memory. For this reason we need the more memory than that of the calculations at zero temperature, or that of the 
calculations of the specific heat at finite temperature [39].

Here we propose a use of the stochastic state selection (SSS) method [40] for calculations of the matrix elements in order to weaken the limitation on the computer memory. This method has been proposed and developed by T. Munehisa and Y. Munehisa thirteen years ago [40]-[47]. For the spin-1/2 antiferromagnet, the number of the basis states amounts to $2^{N}$ with the lattice size $N$, i.e. we need $2^{N}$ coefficients for one state. We select coefficients stochastically so that we force some to be zero and replace others by some finite values. The result from one sampling is not correct, but we can obtain the correct value after making the statistical average. Applying the SSS method we can drastically reduce the number of the basis states with non-zero coefficients. Then the limitation from the memory is relaxed. By this method it is possible to calculate the dynamical correlations on the $N=32$ lattice using the moderate computer whose memory is $64 \mathrm{~GB}$. Note that there is no other method so far to precisely calculate the matrix elements by small portions of the whole states.

After numerical examinations of the SSS method in the FTLM, we present results about the Higgs mode at finite temperature. At low temperature we find the Higgs mode, whereas at high temperature we do not find this mode. We estimate two bounds of temperature by the strict and the loose conditions, under which we can find the Higgs mode. Our results on the lattices from $N=20$ to $N=32$ show that the Higgs mode exists at low temperature and it disappears gradually when the temperature becomes large from the lower bound to the higher bound.

Contents of this paper are as follows. In the next section we present a brief description of calculations in the FTLM which will show the reason why a large number of the Lanczos states are necessary. Sections 3 and 4 are devoted to the SSS method. After explaining the SSS method in Section 3, we present numerical examinations of the SSS method in the calculations of the dynamical correlations in Section 4. Then in Section 5 we calculate the dynamical correlations in the spin-1/2 Heisenberg antiferromagnet on the square lattice in order to find the resonance that is associated with the Higgs mode. The final section is for summary and discussion of this work.

\section{Dynamical Correlations and FTLM}

In this section we give a brief description of the dynamical correlation and of the FTLM we use in our calculations. The dynamical correlation of the operator $\hat{A}$ and $\hat{B}$ is defined by

$$
\begin{aligned}
G(\omega, T) & =\int_{-\infty}^{\infty} d t \operatorname{tr}\left\{e^{-\beta \hat{H}} \hat{A}^{\dagger}(t) \hat{B}(0)\right\} e^{i \omega t} / Z(\beta) \\
& =\int_{-\infty}^{\infty} d t \operatorname{tr}\left\{e^{-\beta \hat{H}} e^{i \hat{H} t} \hat{A}^{\dagger}(0) e^{-i \hat{H} t} \hat{B}(0)\right\} e^{i \omega t} / Z(\beta)
\end{aligned}
$$

Here $Z(\beta)=\operatorname{tr}\left(e^{-\beta \hat{H}}\right)$ and $\beta=1 / T$ is the inverse of the temperature $T$. Using the eigen value $E_{i}$ and the eigen state $\left|\Psi_{i}\right\rangle$ of the Hamiltonian $\hat{H}$, we obtain 


$$
\begin{aligned}
G(\omega, T) & =\sum_{i, j} \int_{-\infty}^{\infty} \mathrm{d} t e^{-\beta E_{i}} e^{i E_{i} t}\left\langle\Psi_{i}\left|\hat{A}^{\dagger}(0)\right| \Psi_{j}\right\rangle e^{-i E_{j} t}\left\langle\Psi_{j}|\hat{B}(0)| \Psi_{i}\right\rangle e^{i \omega t} / Z(\beta) \\
& =\sum_{i, j} e^{-\beta E_{i}} 2 \pi \delta\left(E_{j}-E_{i}-\omega\right)\left\langle\Psi_{i}\left|\hat{A}^{\dagger}(0)\right| \Psi_{j}\right\rangle\left\langle\Psi_{j}|\hat{B}(0)| \Psi_{i}\right\rangle / Z(\beta)
\end{aligned}
$$

It is not possible to calculate every eigen value and every eigen state on the lattice whose size is more than 15 because the number of the matrix element is more than $\left(2^{15}\right)^{2}$. Therefore we approximate the trace calculation using the random state $\left|R_{r}\right\rangle$ [48] [49],

$$
\operatorname{tr}(\hat{O}) \rightarrow \frac{1}{N_{R}} \sum_{r=1}^{N_{R}}\left\langle R_{r}|\hat{O}| R_{r}\right\rangle
$$

We therefore calculate the following $G_{R}\left(\omega, T ; N_{R}\right)$ instead of $G(\omega, T)$,

$$
\begin{aligned}
& G_{R}\left(\omega, T ; N_{R}\right) \\
& =\left\{\frac{1}{N} \sum_{r=1}^{N_{R}} \int_{-\infty}^{\infty} \mathrm{d} t<R_{r}\left|e^{-\beta \hat{H} / 2} e^{i \hat{H} t} \hat{A}^{\dagger}(0) e^{-i \hat{H} t} \hat{B}(0) e^{-\beta \hat{H} / 2}\right| R_{r}>e^{i \omega t}\right\} / Z_{R}\left(\beta ; N_{R}\right)
\end{aligned}
$$

where

$$
Z_{R}\left(\beta ; N_{R}\right)=\frac{1}{N_{R}} \sum_{r=1}^{N_{R}}\left\langle R_{r}\left|e^{-\beta \hat{H}}\right| R_{r}\right\rangle
$$

When $N_{R}$ is infinitely large, $G_{R}\left(\omega, T ; N_{R}\right)$ agrees with $G(\omega, T)$.

In the FTLM, instead of the exact values and the exact states, we use the eigenvalues and the eigen states that are calculated by the set of the Lanczos states. A set of the Lanczos states $\left\{\left|\psi_{k}\right\rangle\right\}(k=1, \cdots, M)$ is generated repeatedly,

$$
\begin{aligned}
\left|\psi_{k+1}\right\rangle & =\left\{\left(\hat{H}-\alpha_{k}\right)\left|\psi_{k}\right\rangle-\beta_{k-1}\left|\psi_{k-1}\right\rangle\right\} / \beta_{k} \\
\alpha_{k} & =\left\langle\psi_{k}|\hat{H}| \psi_{k}\right\rangle \\
\beta_{k} & =\left\{\left\langle\psi_{k}\left|\left(\hat{H}-\alpha_{k}\right)^{2}\right| \psi_{k}\right\rangle-\beta_{k-1}^{2}\right\}^{1 / 2}
\end{aligned}
$$

When $M$ is of order of 100 , it is easy to obtain the eigenvalues of $\hat{H}$.

In the Lanczos method of the FLTM, it is important to choose an appropriate initial state for a good approximation. Therefore in calculations of $G_{R}\left(\omega, T ; N_{R}\right)$ given by (4), the first and second exponents of the Hamiltoniant are separately approximated by the suitable sets. As a result we need two sets of the Lanczos states, which are denoted by $\left\{\left|\psi_{k}\right\rangle_{r}\right\}$ and $\left\{\left|\phi_{k}\right\rangle_{r}\right\}\left(r=1, \cdots, N_{R}\right)$. The first state $\left|\psi_{1}\right\rangle_{r}$ of the set $\left\{\left|\psi_{k}\right\rangle_{r}\right\}$ is given by

$$
\left|\psi_{1}\right\rangle_{r}=e^{-\beta \hat{H} / 2}\left|R_{r}\right\rangle C_{r}
$$

where $C_{r}$ is a normalization factor,

$$
1 / C_{r}^{2}=\left\langle R_{r}\left|e^{-\beta \hat{H}}\right| R_{r}\right\rangle
$$

Similarly the first state $\left|\phi_{1}\right\rangle_{r}$ of the set $\left\{\left|\phi_{k}\right\rangle_{r}\right\}$ is given by

$$
\left|\phi_{1}\right\rangle_{r}=\hat{B}(0) e^{-\beta \hat{H} / 2}\left|R_{r}\right\rangle C_{B r}
$$

where $C_{B r}$ is a normalization factor, 


$$
1 / C_{B r}^{2}=\left\langle R_{r}\left|e^{-\beta \hat{H} / 2} \hat{B}^{\dagger}(0) \hat{B}(0) e^{-\beta \hat{H} / 2}\right| R_{r}\right\rangle
$$

Let us denote the $i$-th eigen value of the Hamiltonian $(i=1,2, \cdots, M)$ in the set $\left\{\left|\psi_{k}\right\rangle_{r}\right\}$ by $E_{\psi, i}^{(r)}$ and the eigen state by $\left|E_{\psi, i}\right\rangle_{r}$, while in the set $\left\{\left|\phi_{k}\right\rangle_{r}\right\}$ they are denoted by $E_{\phi, i}^{(r)}$ and by $\left|E_{\phi, i}\right\rangle_{r}$. The first exponential operator $e^{i \hat{H} t}$ is replaced by the set $\left\{\left|\psi_{k}\right\rangle_{r}\right\}$,

$$
e^{i \hat{H} t} \rightarrow \sum_{i=1}^{M}\left|E_{\psi, i}\right\rangle_{r} e^{i E_{\psi, i}^{(r) t}}\left\langle E_{\psi, i}\right|
$$

Similarly the second exponential operator $e^{-i \hat{H} t}$ is replaced by the set $\left\{\left|\phi_{k}\right\rangle_{r}\right\}$

$$
e^{-i \hat{H} t} \rightarrow \sum_{j=1}^{M}\left|E_{\phi, j}\right\rangle_{r} e^{-i E_{\phi, j}^{(r)} t}\left\langle E_{\phi, j}\right|
$$

When we use these eigenvalues and eigen states, we obtain the following expression for $G_{R}\left(\omega, T ; N_{R}\right)$,

$$
\begin{aligned}
& G_{R}\left(\omega, T ; N_{R}\right) \\
= & \left\{\frac{1}{N_{R}} \sum_{r=1}^{N_{R}} \sum_{i, j=1}^{M} \int_{-\infty}^{\infty} \mathrm{d} t C_{r}^{-1} e^{i E_{\psi, i}^{(r)}}{ }_{r}\left\langle\psi_{1} \mid E_{\psi, i}\right\rangle_{r}\right. \\
& \left.\times{ }_{r}\left\langle E_{\psi, i}\left|\hat{A}^{\dagger}(0)\right| E_{\phi, j}\right\rangle_{r} e^{-i E_{\phi, j}^{(r) t}}\left\langle E_{r, j} \mid \phi_{1}\right\rangle_{r} C_{B r}^{-1} e^{i \omega t}\right\} / Z_{R}\left(\beta ; N_{R}\right) \\
= & \left\{\frac{1}{N_{R}} \sum_{r=1}^{N_{R}} \sum_{i, j=1}^{M} C_{r}^{-1}{ }_{r}\left\langle\psi_{1} \mid E_{\psi, i}\right\rangle_{r} r_{r}\left\langle E_{\psi, i}\left|\hat{A}^{\dagger}(0)\right| E_{\phi, j}\right\rangle_{r}\left\langle E_{\phi, j} \mid \phi_{1}\right\rangle_{r} C_{B r}^{-1}\right. \\
& \left.\times 2 \pi \delta\left(E_{\phi, j}^{(r)}-E_{\psi, i}^{(r)}-\omega\right)\right\} / Z_{R}\left(\beta ; N_{R}\right)
\end{aligned}
$$

We cannot calculate the $\delta$-function on the finite lattice because of the discrete eigenvalues. Instead of this singular function, therefore, we use a regular function with a parameter $\varepsilon$,

$$
2 \pi \delta(x) \rightarrow \frac{2 \varepsilon}{x^{2}+\varepsilon^{2}}
$$

Based on the discussions in [29], we make $\varepsilon$ a moderate value in order to examine peaks of $G_{R}\left(\omega, T ; N_{R}\right)$ that are made by several eigen states. Then we obtain the following expression

$$
\begin{aligned}
& G_{R}\left(\omega, T ; N_{R}\right) \\
& =\left\{\frac{1}{N_{R}} \sum_{r=1}^{N_{R}} \sum_{i, j=1}^{M} C_{r}^{-1}{ }_{r}\left\langle\psi_{1} \mid E_{\psi, i}\right\rangle_{r}{ }_{r}\left\langle E_{\psi, i}\left|\hat{A}^{\dagger}(0)\right| E_{\phi, j}\right\rangle_{r_{r}}\left\langle E_{\phi, j} \mid \phi_{1}\right\rangle_{r} C_{B r}^{-1}\right. \\
& \left.\times \frac{2 \varepsilon}{\left[\left(E_{\phi, j}^{(r)}-E_{\psi, i}^{(r)}-\omega\right)^{2}+\varepsilon^{2}\right]}\right\} / Z_{R}\left(\beta ; N_{R}\right)
\end{aligned}
$$

\section{Stochastic State Selection Method}

In this section we briefly describe the stochastic state selection method [40]. A state $|\psi\rangle$ is given by a set of a basis states $\{|i\rangle\}\left(i=1, \cdots, N_{V}\right)$, 


$$
|\psi\rangle=\sum_{i}|i\rangle c_{i}, \quad\left(c_{i} \neq 0\right)
$$

Let us consider a probability variable $\eta$ for a parameter $a \geq 1$, which is defined by $\eta=a$ with the probability $P(\eta=a)=1 / a$ and $\eta=0$ with the probability $P(\eta=0)=1-1 / a$. The average of this variable is one, i.e.

$\langle\langle\eta\rangle\rangle=a \cdot P(\eta=a)+0 \cdot P(\eta=0)=a \cdot 1 / a+0 \cdot(1-1 / a)=1$. Here $\langle\langle\cdot\rangle\rangle$ is the statistical average. Note that $\left\langle\left\langle\eta^{2}\right\rangle\right\rangle=a$. We introduce a state $|\psi\rangle_{\eta}$ to approximate the state $|\psi\rangle$,

$$
|\psi\rangle_{\eta}=\sum_{i}|i\rangle c_{i} \eta_{c, i}
$$

where $\eta_{c, i}$ is a probability variable generated with $a_{c, i}=\max \left(\varepsilon_{S} /\left|c_{i}\right|, 1\right)$. Here $\varepsilon_{S}$ is a parameter to control the accuracy of $|\psi\rangle_{\eta}$ and the number of the selected states in the SSS method. When we make the statistical average of $|\psi\rangle_{\eta}$, we obtain the correct state,

$$
\left.\left\langle\langle\mid \psi\rangle_{\eta}\right\rangle\right\rangle=\sum_{i}|i\rangle c_{i}\left\langle\left\langle\eta_{c, i}\right\rangle\right\rangle=\sum_{i}|i\rangle c_{i}=|\psi\rangle
$$

The statistical average of number $N_{\psi}$ of basis states with non-zero coefficients in the sampling is given by

$$
\left\langle\left\langle N_{\psi}\right\rangle\right\rangle=\sum_{i}\left\{1 \cdot P\left(\eta_{c, i}=a_{c, i}\right)+0 \cdot P\left(\eta_{c, i}=0\right)\right\}=\sum_{i} 1 / a_{c, i}=\sum_{i} \min \left(\left|c_{i}\right| / \varepsilon_{S}, 1\right)
$$

Next we consider an inner product $\langle\psi \mid \phi\rangle=\sum_{i} c_{i}^{*} b_{i}$ of two states $|\psi\rangle$ and $|\phi\rangle=\sum_{i}|i\rangle b_{i}\left(b_{i} \neq 0\right)$. Using $|\psi\rangle_{\eta}$ in (17) and

$$
|\phi\rangle_{\eta}=\sum_{i}|i\rangle b_{i} \eta_{b, i}
$$

where $\eta_{b, i}$ is a probability variable generated with $a_{b, i}=\max \left(\varepsilon_{s} /\left|b_{i}\right|, 1\right)$, we calculate the inner product,

$$
{ }_{\eta}\langle\psi \mid \phi\rangle_{\eta}=\sum_{i} c_{i}^{*} b_{i} \eta_{c, i} \eta_{b, i}
$$

If we make the statistical average of the inner product we obtain the correct value,

$$
\left.\left\langle{ }_{\eta}\langle\psi \mid \phi\rangle_{\eta}\right\rangle\right\rangle=\sum_{i} c_{i}^{*} b_{i}\left\langle\left\langle\eta_{c, i} \eta_{b, i}\right\rangle\right\rangle=\sum_{i} c_{i}^{*} b_{i}\left\langle\left\langle\eta_{c, i}\right\rangle\right\rangle\left\langle\left\langle\eta_{b, i}\right\rangle\right\rangle=\langle\psi \mid \phi\rangle
$$

In our calculation the statistical average $\langle\langle\cdot\rangle\rangle$ is replaced by a sample average with a sample number $N_{s m}$,

$$
\langle\langle A\rangle\rangle \rightarrow \frac{1}{N_{s m}} \sum_{k=1}^{N_{s m}} A_{k}
$$

where $A_{k}$ is a value of $A$ in one sampling. When $N_{s m}$ becomes infinitely large, the sample average agrees with the statistical average.

When $\varepsilon_{S}$ becomes large the number of the non-zero $\eta$ decreases, but the larger $N_{s m}$ is necessary for more accurate value. In order to estimate the required $N_{s m}$, we calculate a variance $\sigma^{2}$ of the inner product, because the accuracy of the sample average is given by $\sigma / \sqrt{N_{s m}}$. 


$$
\begin{aligned}
\sigma^{2}= & \left\langle\left\langle\left({ }_{\eta}\langle\psi \mid \phi\rangle_{\eta}\right)^{2}\right\rangle\right\rangle-\left(\left\langle\left\langle{ }_{\eta}\langle\psi \mid \phi\rangle_{\eta}\right\rangle\right\rangle\right)^{2} \\
= & \sum_{i} \sum_{j}\left(c_{i}^{*} b_{i}\right)\left(c_{j}^{*} b_{j}\right)\left\langle\left\langle\eta_{c, i} \eta_{b, i} \eta_{c, j} \eta_{b, j}\right\rangle\right\rangle-\left\{\sum_{i} c_{i}^{*} b_{i}\right\}^{2} \\
= & \sum_{i \neq j}\left(c_{i}^{*} b_{i}\right)\left(c_{j}^{*} b_{j}\right)\left\langle\left\langle\eta_{c, i}\right\rangle\right\rangle\left\langle\left\langle\eta_{b, i}\right\rangle\right\rangle\left\langle\left\langle\eta_{c, j}\right\rangle\right\rangle\left\langle\left\langle\eta_{b, j}\right\rangle\right\rangle \\
& +\sum_{i}\left(c_{i}^{*} b_{i}\right)^{2}\left\langle\left\langle\eta_{c, i}^{2}\right\rangle\right\rangle\left\langle\left\langle\left\langle\eta_{b, i}^{2}\right\rangle\right\rangle-\left\{\sum_{i} c_{i}^{*} b_{i}\right\}^{2}\right. \\
= & \sum_{i \neq j}\left(c_{i}^{*} b_{i}\right)\left(c_{j}^{*} b_{j}\right)+\sum_{i}\left(c_{i}^{*} b_{i}\right)^{2} a_{c, i} a_{b, i}-\left\{\sum_{i \neq j}\left(c_{i}^{*} b_{i}\right)\left(c_{j}^{*} b_{j}\right)+\sum_{i}\left(c_{i}^{*} b_{i}\right)^{2}\right\} \\
= & \sum_{i}\left(c_{i}^{*} b_{i}\right)^{2}\left\{\max \left(\varepsilon_{S} /\left|c_{i}\right|, 1\right) \cdot \max \left(\varepsilon_{S} /\left|b_{i}\right|, 1\right)-1\right\}
\end{aligned}
$$

For quantitative discussions let us assume that $\varepsilon_{S} \gg 1 / \sqrt{N_{V}}$ and $c_{i}=b_{i}=1 / \sqrt{N_{V}}$ for all $i$. Then we obtain

$$
\sigma^{2}=\sum_{i}\left(1 / \sqrt{N_{V}}\right)^{4}\left\{\left(\varepsilon_{S} \sqrt{N_{V}}\right)^{2}-1\right\} \approx \varepsilon_{S}^{2}
$$

and

$$
\left\langle\left\langle N_{\psi}\right\rangle\right\rangle=\sum_{i} \min \left\{\left(\left(1 / \sqrt{N_{V}}\right) / \varepsilon_{S}\right), 1\right\}=1 /\left(\sqrt{N_{V}} \varepsilon_{S}\right) \sum_{i=1}^{N_{V}} 1=\sqrt{N_{V}} / \varepsilon_{S}
$$

If $N_{V}=10^{8}$ and $\varepsilon_{S}=0.01$, we have $\sigma^{2}=0.0001$ and $\left\langle\left\langle N_{\psi}\right\rangle\right\rangle=10^{6}$. Thus we can obtain the inner product with satisfyingly small $\sigma$ even if $\left\langle\left\langle N_{\psi}\right\rangle\right\rangle \ll N_{V}$. In this case we can obtain the accuracy of $0.01 / \sqrt{N_{s m}}$ for the inner product $\langle\psi \mid \phi\rangle$.

\section{Numerical Examinations of SSS Method}

In this section we present numerical examinations of the SSS method in the calculations of the dynamical correlations. Our model is the spin-1/2 Heisenberg antiferromagnet on the square lattice. The Hamiltonian is given by

$$
\hat{H}=\sum_{(i, j)}\left\{\hat{s}_{i}^{x} \hat{s}_{j}^{x}+\hat{s}_{i}^{y} \hat{s}_{j}^{y}+\hat{s}_{i}^{x} \hat{s}_{j}^{z}\right\}
$$

where $\hat{s}_{i}^{l}(l=x, y, z)$ is a spin operator on a site $i(i=1, \cdots, N)$ and $(i, j)$ denotes the nearest neighbor pair on the square lattice. The $z$-component $\hat{s}_{i}^{z}$ is a diagonal matrix, i.e. the basis state is defined by $\left|s_{1}, s_{2}, \cdots, s_{N}\right\rangle$ where $s_{i}=1 / 2$ or $s_{i}=-1 / 2$. Since our purpose is to investigate the Higgs mode, the operator $\hat{A}(0)$ and $\hat{B}(0)$ are the two spin operators. Namely $\hat{A}(0)=\hat{B}(0)=\hat{s}^{\mathrm{z}}(\boldsymbol{k}) \hat{s}^{\mathrm{z}}(-\boldsymbol{k}) \quad[29]$, where

$$
\hat{s}^{z}(\boldsymbol{k})=\frac{1}{\sqrt{N}} \sum_{i=1}^{N} e^{-i k_{i}} \hat{s}_{i}^{z}
$$

denoting the location of site $i$ by $\boldsymbol{r}_{i}=\left(x_{i}, y_{i}\right)$.

In the calculations of the dynamical correlations at finite temperature, we apply the SSS method to two sets of the Lanczos states, $\left\{\left|\psi_{i}\right\rangle_{r}\right\}$ and $\left\{\left|\phi_{i}\right\rangle_{r}\right\}$. In the calculations of ${ }_{r}\left\langle E_{\psi, i}\left|\hat{A}^{\dagger}(0)\right| E_{\phi, j}\right\rangle_{r}$, we should keep $2 \times M \times 2^{N}$ coef- 
ficients on the lattice of the size $N$ without the SSS method. When we apply the SSS method, on the other hand, we need to keep $\sim 2 \times M \times N_{\psi}$ coefficients instead. As we have discussed in section 3 we can impose $N_{\psi}$ which is much smaller than $2^{N}$.

Let us examine the accuracy by the SSS method then. For this examination we use a state $|F\rangle$ instead of $\left|R_{r}\right\rangle\left(r=1, \cdots, N_{R}\right)$ and calculate a correlation.

$$
G_{F}(\omega, T)=\int_{-\infty}^{\infty} \mathrm{d} t\left\langle F\left|e^{-\beta \hat{H} / 2} e^{i \hat{H} t} \hat{A}^{\dagger}(0) e^{-i \hat{H} t} \hat{B}(0) e^{-\beta \hat{H} / 2}\right| F\right\rangle e^{i \omega t} /\left\langle F\left|e^{-\beta \hat{H}}\right| F\right\rangle
$$

instead of $G_{R}\left(\omega, T ; N_{R}\right)$ in (4). Following the procedure of having (15) from (4) in the section 2 , we obtain

$$
\begin{aligned}
G_{F}(\omega, T)= & \sum_{i, j=1}^{M}\left\{C_{F}^{-1}{ }_{F}\left\langle\psi_{1} \mid E_{\psi, i}\right\rangle_{F F}\left\langle E_{\psi, i}\left|\hat{A}^{\dagger}(0)\right| E_{\phi, j}\right\rangle_{F F}\left\langle E_{\phi, j} \mid \phi_{1}\right\rangle_{F} C_{B F}^{-1}\right. \\
& \left.\times \frac{2 \varepsilon}{\left[\left(E_{\phi, j}^{(F)}-E_{\psi, i}^{(F)}-\omega\right)^{2}+\varepsilon^{2}\right]}\right\} /\left\langle F\left|e^{-\beta \hat{H}}\right| F\right\rangle
\end{aligned}
$$

where $C_{F}$ and $C_{B F}$ are normalization factors,

$$
\begin{gathered}
1 / C_{F}^{2}=\left\langle F\left|e^{-\beta \hat{H}}\right| F\right\rangle \\
1 / C_{B F}^{2}=\left\langle F\left|e^{-B \hat{H} / 2} \hat{B}^{\dagger}(0) \hat{B}(0) e^{-B \hat{H} / 2}\right| F\right\rangle
\end{gathered}
$$

Note that, from $\left|E_{\psi, i}\right\rangle_{F}=\sum_{k}\left|\psi_{k}\right\rangle_{F} a_{i, k}^{(F)}$ and $\left|E_{\phi, i}\right\rangle_{F}=\sum_{k}\left|\phi_{k}\right\rangle_{F} b_{i, k}^{(F)}$, we have

$$
\begin{gathered}
\left\langle\psi_{1} \mid E_{\psi, i}\right\rangle_{F}=a_{i, 1}^{(F)} \\
\left\langle E_{\phi, j} \mid \phi_{1}\right\rangle_{F}=b_{j, 1}^{\dagger(F)} \\
\left\langle E_{\psi, i}\left|\hat{A}^{\dagger}(0)\right| E_{\phi, j}\right\rangle_{F}=\sum_{k_{1}, k_{2}=1}^{M} a_{i, k_{1}}^{\dagger(F)} b_{j, k_{2}}^{(F)}\left\langle\psi_{k_{1}}\left|\hat{A}^{\dagger}(0)\right| \phi_{k_{2}}\right\rangle_{F}
\end{gathered}
$$

We apply the SSS method to $\left|\psi_{i}\right\rangle_{F}$ and $\left|\phi_{j}\right\rangle_{F}$ in order to obtain the SSS states $\left|\psi_{i}\right\rangle_{F, \eta}$ and $\left|\phi_{j}\right\rangle_{F, \eta}$. Using these states we define $G_{F, \eta}\left(\omega, T ; \varepsilon_{S}, N_{s m}\right)$ $=C_{F}^{-1} C_{B F}^{-1} \sum_{i, j=1}^{M}\left\{a_{i, 1}^{(F)} b_{j, 1}^{\dagger(F)} \sum_{k_{1}, k_{2}=1}^{M} a_{i, k_{1}}^{\dagger(F)} b_{j, k_{2}}^{(F)}\right.$

$$
\left.\times \frac{1}{N_{s m}} \sum_{n=1}^{N_{s m}}\left[{ }_{F, \eta}\left\langle\psi_{k_{1}}\left|\hat{A}^{\dagger}(0)\right| \phi_{k_{2}}\right\rangle_{F, \eta}\right]_{n} \frac{2 \varepsilon}{\left[\left(E_{\phi, j}^{(F)}-E_{\psi, i}^{(F)}-\omega\right)^{2}+\varepsilon^{2}\right]}\right\} /\left\langle F\left|e^{-\beta \hat{H}}\right| F\right\rangle
$$

Here $\left[{ }_{F, \eta}\left\langle\psi_{k_{1}}\left|\hat{A}^{\dagger}(0)\right| \phi_{k_{2}}\right\rangle_{F, \eta}\right]_{n}$ denotes ${ }_{F, \eta}\left\langle\psi_{k_{1}}\left|\hat{A}^{\dagger}(0)\right| \phi_{k_{2}}\right\rangle_{F, \eta}$ of the $n$-th SSS sampling. When $N_{s m}$ is infinitely large $G_{F, \eta}\left(\omega, T ; \varepsilon_{S}, N_{s m}\right)$ agrees with $G_{F}(\omega, T)$.

As discussed in section 3, we have the parameter $\varepsilon_{S}$ in the SSS method. When $\varepsilon_{S}$ is small the accuracy is high, but the number of the basis states to 
be kept is large. We would like to examine the accuracy and the cost in calculations of $G_{F, \eta}\left(\omega, T ; \varepsilon_{S}, N_{s m}\right)$. In Figure 1, we show our numerical results of $G_{F, \eta}\left(\omega, T ; \varepsilon_{S}, N_{s m}\right)$ on the lattice $N=20$ with $1 / T=1.60, \quad \boldsymbol{k}=(2 \pi / 5, \pi / 5)$ and $\varepsilon=0.5$ as a function of $\omega$. In this figure we plot the difference $D_{F}\left(\omega ; \varepsilon_{S}, N_{s m}\right)$ given by

$$
D_{F}\left(\omega ; \varepsilon_{S}, N_{s m}\right)=G_{F, \eta}\left(\omega, T ; \varepsilon_{S}, N_{s m}\right)-G_{F}(\omega, T)
$$

The error bar is the statistical error of $G_{F, \eta}\left(\omega, T ; \varepsilon_{S}, N_{s m}\right)$. Compared with the black data $\left(\varepsilon_{S}=0.08, N_{s m}=40\right)$, the green data $\left(\varepsilon_{s}=0.08, N_{s m}=720\right)$, are closer to zero. In comparison with the black data and the red data:

$\left(\varepsilon_{S}=0.02, N_{s m}=40\right)$, the latters are closer to zero. The average numbers of the basis states with non-zero coefficients in the SSS method are 9500 with $\varepsilon_{S}=0.08$ and $\sim 37000$ with $\varepsilon_{S}=0.02$. In section 3 we have argued that the average number of the basis states with non-zero coefficients is drastically reduced to be of order of $\sqrt{N_{V}} / \varepsilon_{S}$ by the SSS method. We see that the measured values are a little less than $\sqrt{N_{V}} / \varepsilon_{S}$, which are 12500 for $\varepsilon_{S}=0.08$ and 51200 for $\varepsilon_{S}=0.02$.

As for the accuracy of sample average, we have discussed that it would be proportional to $\varepsilon_{S} / \sqrt{N_{s m}}$. Figure 1 shows that the error around $\omega=3.0$ is $\sim 0.04$ in the black data, $\sim 0.01$ in the red data and $\sim 0.01$ in the green data. Since $\left\{\varepsilon_{S} / \sqrt{N_{s m}}\right.$ (black data) $\} /\left\{\varepsilon_{S} / \sqrt{N_{s m}}\right.$ (red data) $\}=4 \sim 0.04 / 0.01$ and $\left\{\varepsilon_{S} / \sqrt{N_{s m}}\right.$ (black data) $\} /\left\{\varepsilon_{S} / \sqrt{N_{s m}}\right.$ (green data) $\}=\sqrt{18} \sim 0.04 / 0.01$, these results support the discussion in section 3 .

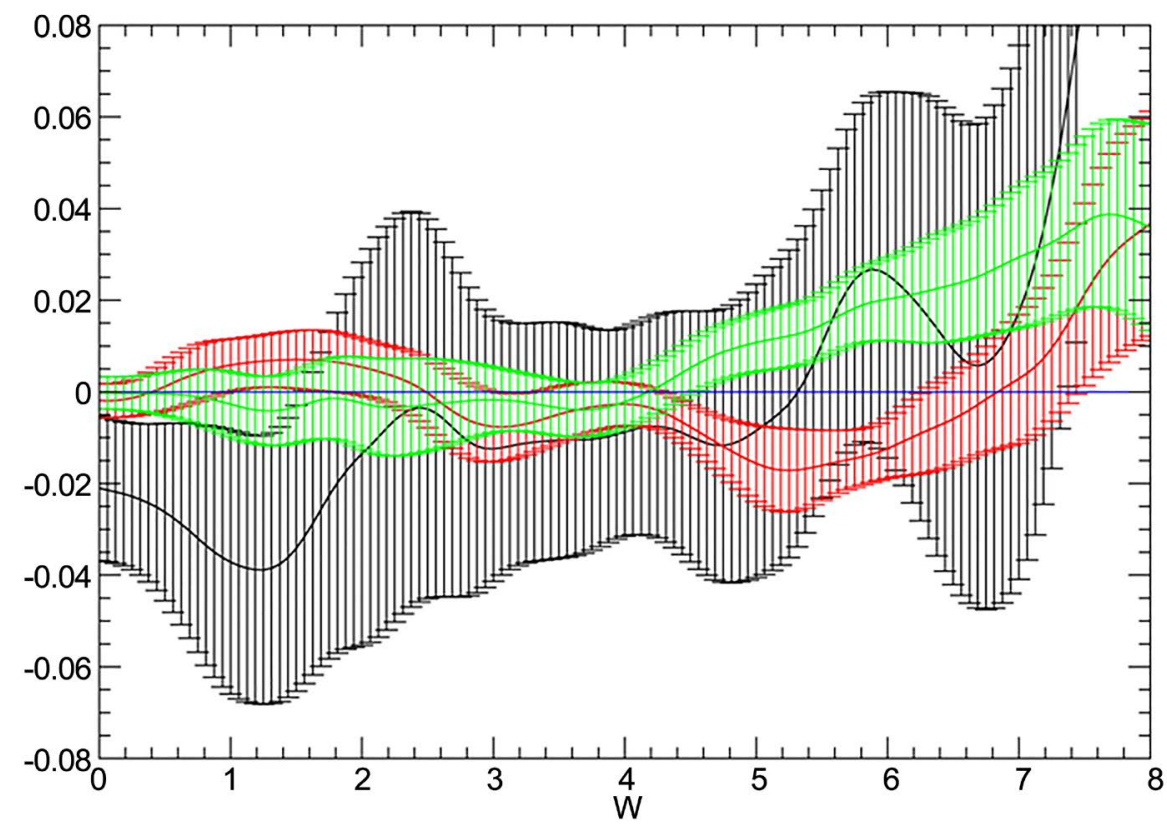

Figure 1. The difference $D_{F}\left(\omega ; \varepsilon_{S}, N_{s m}\right)$ defined in (37) in the SSS method on the $N=20$ lattice for the fixed state with $1 / T=1.60$ and $\boldsymbol{k}=(2 \pi / 5, \pi / 5)$. They are plotted as a function of $\omega$. Parameters $\varepsilon_{s}$ and $N_{s m}$ are 0.08 and 40 for the black data, 0.02 and 40 for the red data and 0.08 and 720 for the green data, respectively. 
In order to examine $G_{R}\left(\omega, T ; N_{R}\right)$ using the SSS method, we define $G_{R, \eta}\left(\omega, T ; N_{R}, \varepsilon_{S}\right)$ by

$$
\begin{aligned}
& G_{R, \eta}\left(\omega, T ; N_{R}, \varepsilon_{S}\right) \\
& =\left\{\frac{1}{N_{R}} \sum_{r=1}^{N_{R}} C_{F}^{-1} C_{B r}^{-1} \sum_{i, j=1}^{M} a_{i, 1}^{(r)} b_{j, 1}^{\dagger(r)} \sum_{k_{1}, k_{2}=1}^{M} a_{i, k_{1}}^{\dagger \dagger(r)} b_{j, k_{2}}^{(r)}\right. \\
& \left.\times_{r, \eta}\left\langle\psi_{k_{1}}\left|\hat{A}^{\dagger}(0)\right| \phi_{k_{2}}\right\rangle_{r, \eta} \overline{\left[\left(E_{\phi, j}^{(r)}-E_{\psi, i}^{(r)}-\omega\right)^{2}+\varepsilon^{2}\right]}\right\} / Z_{R}\left(\beta ; N_{R}\right) \\
& Z_{R}\left(\beta ; N_{R}\right)=\frac{1}{N_{R}} \sum_{r=1}^{N_{R}}\left\langle R_{r}\left|e^{-\beta \hat{H}}\right| R_{r}\right\rangle
\end{aligned}
$$

Note that here we set $N_{s m}=1$ because one sampling for each random set will be enough when $N_{R}$ is large. Further discussion on this point will be given in the final section.

Now we would like to examine the difference between $G_{R, \eta}\left(\omega, T ; N_{R}, \varepsilon_{S}\right)$ and $G(\omega, T)$ on the $N=20$ lattice. Even on this small lattice, however, it is difficult to obtain $G(\omega, T)$ because we need all eigenvalues and eigen states for the correlations. We therefore use $G_{R}\left(\omega, T ; N_{R}=640\right)$ here instead of $G(\omega, T)$. In Figure 2 we plot $D\left(N_{R}, \varepsilon_{S}\right)$ which is defined by

$$
D\left(N_{R}, \varepsilon_{S}\right)=\sum_{i=0}^{N_{\omega}}\left|G_{R, \eta}\left(\omega_{i}, T ; N_{R}, \varepsilon_{S}\right) / G_{R}\left(\omega_{i}, T ; N_{R}=640\right)-1\right| / N_{\omega}
$$

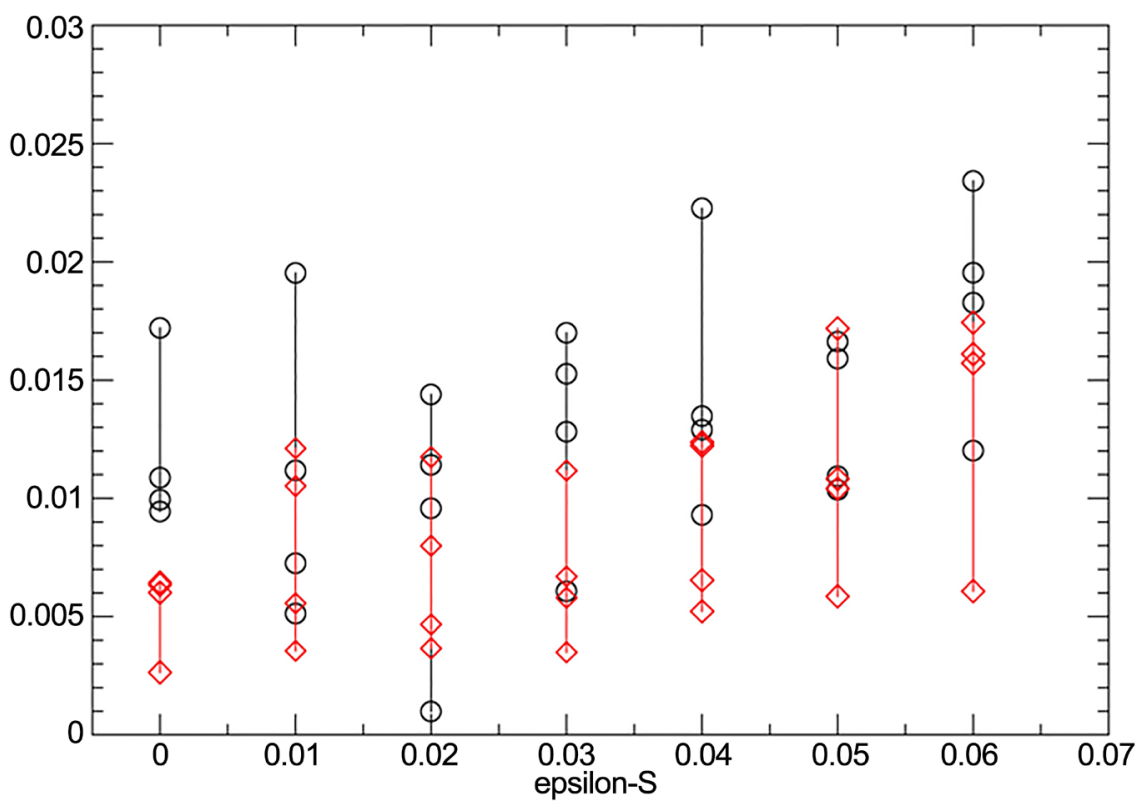

Figure 2. The difference $D\left(N_{R}, \varepsilon_{S}\right)$ defined in (40) in the SSS method on the $N=20$ lattice with $1 / T=1.60$ and $\boldsymbol{k}=(2 \pi / 5, \pi / 5)$. For each $\varepsilon_{S}$ we present four $D\left(N_{R}, \varepsilon_{S}\right)$ calculated by four sets of $\left\{\left|R_{1}\right\rangle,\left|R_{2}\right\rangle, \cdots,\left|R_{N_{R}}\right\rangle\right\}$. They are plotted by black circles $\left(N_{R}=64\right)$ and by red circles $\left(N_{R}=256\right)$. Results of $\varepsilon_{S}=0$ are obtained without using the SSS method. 
where $\omega_{i}=i \times \Delta \omega, \Delta \omega=0.0625$, and $N_{\omega}=120$. In order to examine the fluctuation of $G_{R, \eta}\left(\omega, T ; N_{R}, \varepsilon_{S}\right)$ due to the sampling, we carry out calculations with four sets of $\left\{\left|R_{1}\right\rangle,\left|R_{2}\right\rangle, \cdots,\left|R_{N_{R}}\right\rangle\right\}$ for each $\varepsilon_{S}$. The observed four $D\left(N_{R}, \varepsilon_{S}\right)$ are plotted by the black circles $\left(N_{R}=64\right)$ and the red diamonds $\left(N_{R}=256\right)$. In Figure 2 we also plot results for $D\left(N_{R}, \varepsilon_{S}=0\right)$, which are obtained without using the SSS method. Note that they should give us the minimum of the accuracy for $D\left(N_{R}, \varepsilon_{S} \neq 0\right)$. For both cases of $N_{R}=64$ and $N_{R}=256$ we observe that $D\left(N_{R}, \varepsilon_{S} \neq 0\right)$ and $D\left(N_{R}, \varepsilon_{S}=0\right)$ are almost the same order for any value of $\varepsilon_{S}$ we employed. This fact implies that in calculations of the dynamical correlations using the SSS method we do not need more number of the sampling compared to that without the SSS. By these examinations we conclude that we can apply the SSS method to calculations of the dynamical correlations.

\section{Higgs Mode}

The most important purpose of this paper is the numerical verification of the Higgs mode in the quantum spin systems at finite temperature. In this section we would like to show it by calculating the dynamical correlations in the spin$1 / 2$ Heisenberg antiferromagnet on the square lattice. Since the Higgs mode is the excited state and couples to the two Goldstone-Nambu modes, we have to calculate the dynamical correlations of the two operators that contain the Goldstone-Nambu modes. In the Heisenberg antiferromagnet the spin operators contain these modes. Therefore we calculate the following dynamical correlation, $G_{R, \eta}\left(\omega, T ; N_{R}, \varepsilon_{S}\right)$ in (39) with the two spin operators $\hat{A}(0)=\hat{B}(0)=\hat{s}^{z}(\boldsymbol{k}) \hat{s}^{\mathrm{Z}}(-\boldsymbol{k})$.

In order to obtain stable results at any temperature, we employ the Chebyshev polynomial expansion [39] for the calculation of $e^{-\beta \hat{H} / 2}\left|R_{r}\right\rangle$ in (7) and (9),

$$
e^{-\beta \hat{H} / 2}\left|R_{r}\right\rangle=\sum_{k=1}^{N c} p_{k}(\beta / 2) T_{k}\left(\hat{H} / h_{c}\right)\left|R_{r}\right\rangle
$$

with the $k$-th Chebyshev polynomial $T_{k}(x)$ and the $k$-th coefficient $p_{k}$.

Before presenting our results we comment on parameters in our calculations, which we summarize in Table 1.

Table 1. Parameters of calculations; the symbol and the range in our calculations.

\begin{tabular}{cc}
\hline$/ T$ & $0.80-10.0$ \\
$\omega$ & $0.0-8.0$ \\
$N$ & $20-32$ \\
$\boldsymbol{k}$ & Minimum wave vector on the lattice \\
$N_{c}$ & $20-100$ \\
$h_{c}$ & $15-25$ \\
$\varepsilon$ & 0.5 \\
$M$ & $40-50$ \\
$\varepsilon_{S}$ & $0.01-0.04$ \\
$N_{R}$ & $20-160$ \\
\hline
\end{tabular}


The inverse temperature $\beta=1 / T$ and the energy $\omega$ are the physical quantities.

The lattice size $N$ is restricted because of the exact diagonalization approach.

In our work, we calculate on the lattices of the size $20 \leq N \leq 32$. For the periodic boundary condition we have two edge vectors $\left(l_{11}, l_{12}\right)$ and $\left(l_{21}, l_{22}\right)$. Since we impose the $\pi / 2$ rotational symmetry to the Hamiltonian, the edge vector $\left(l_{21}, l_{22}\right)$ is given by $\left(-l_{12}, l_{11}\right)$ and the lattice size is given by $N=l_{11}^{2}+l_{12}^{2}$. Note that these edge vectors are defined uniquely for a given lattice size $N$ except for an accidental case $N=25$. In this exceptional case we distinguish two different $N=25$ lattices by $25 a$ and $25 b$. For the lattices of $N=20,25 a, 25 b, 26,29$ and 32 , the edge vector $\left(l_{11}, l_{12}\right)$ are $(4,2),(5,0),(4,3)$, $(5,1),(5,2)$ and $(4,4)$, respectively.

The wave vector $\boldsymbol{k}$ is the non-zero wave vector of the lowest magnitude on the each lattice. For the lattices $N=20,25 a, 25 b, 26,29$ and 32, they are $(2 \pi / 5, \pi / 5),(2 \pi / 5,0),(8 \pi / 25,6 \pi / 25),(5 \pi / 13, \pi / 13),(10 \pi / 29,4 \pi / 29)$ and $(\pi / 4, \pi / 4)$.

The parameters $N_{c}$ and $h_{c}$ control the accuracy of the Chebyshev polynomial expansion. They are determined by the request that the precision is of order $10^{-12}$. As a result they depend on values of $N$ and $T$.

On the parameter $\varepsilon$ in (14), we have presented the careful discussion in the previous work [29]. This discussion has showed that $\varepsilon=0.5$ is most suitable. Therefore we use this value for $\varepsilon$.

As for the number $M$ of the Lanczos states we fix it to be 50 following the discussion of the previous work [29] and the preliminary study. In the $N=32$ lattice we use $M=40$ in order to reduce a huge calculation time.

We apply the SSS method to calculations for $N \geq 25$. The parameter $\varepsilon_{S}$ is 0.01 for $N=25 a, 25 b, 26$ and 29 , and is 0.04 for $N=32$.

The sampling number of the random states $N_{R}$ is determined by requiring that the relative precision of our calculations on the dynamical correlations is $5 \%$.

In Figure 3 we present the dynamical correlations $G_{R, \eta}\left(\omega, T ; N_{R}, \varepsilon_{S}\right)$ on the $N=29$ lattice as a function of $\omega$. At the low temperatures $1 / T=10.0,6.4$ and 3.2 we find the broad peaks clearly, as expected. These broad peaks could be the Higgs mode which has been found at $T=0$ in the previous work [29]. On the other hand, at the high temperature $1 / T=1.2$ we cannot find any peak that is relevant with the Higgs mode. In order to confirm that we find the broad peak at the low $T$ in the contrast to no broad peak at high $T$, we plot the dynamical correlations at the low $T$ and those at the high $T$ on various lattices in Figure 4, where the correlations at $1 / T=3.2$ and those at $1 / T=1.2$ are shown. The results in Figure 4 support our arguments.

What we are interested in is the shape of the dynamical correlation around the broad peak. Since the absolute value of the correlation strongly depends on $T$, we introduce a normalized correlation 


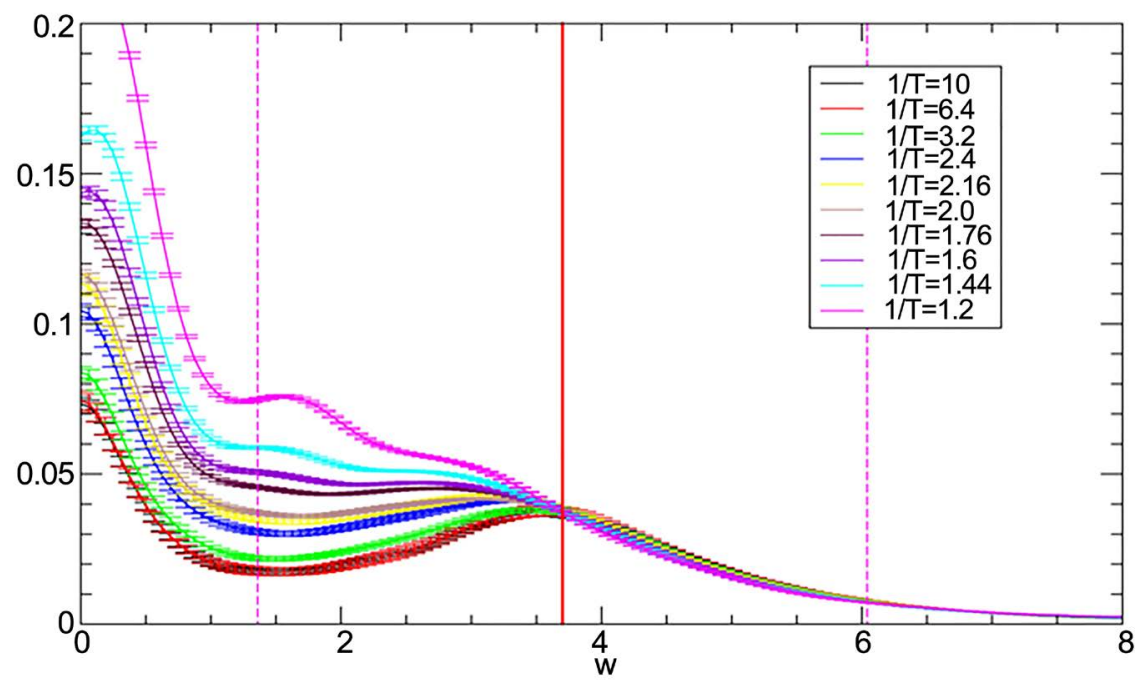

Figure 3. The dynamical correlations $G_{R, \eta}\left(\omega, T ; N_{R}, \varepsilon_{S}\right)$ on the $N=29$ lattice for various values of $T$. They are plotted as a function of $\omega$. The error bars are the statistical errors. The vertical red solid-line shows the value of $\omega_{c}$. The vertical violet dashed-lines show the value of $\omega_{c} \pm 2 \Gamma$.

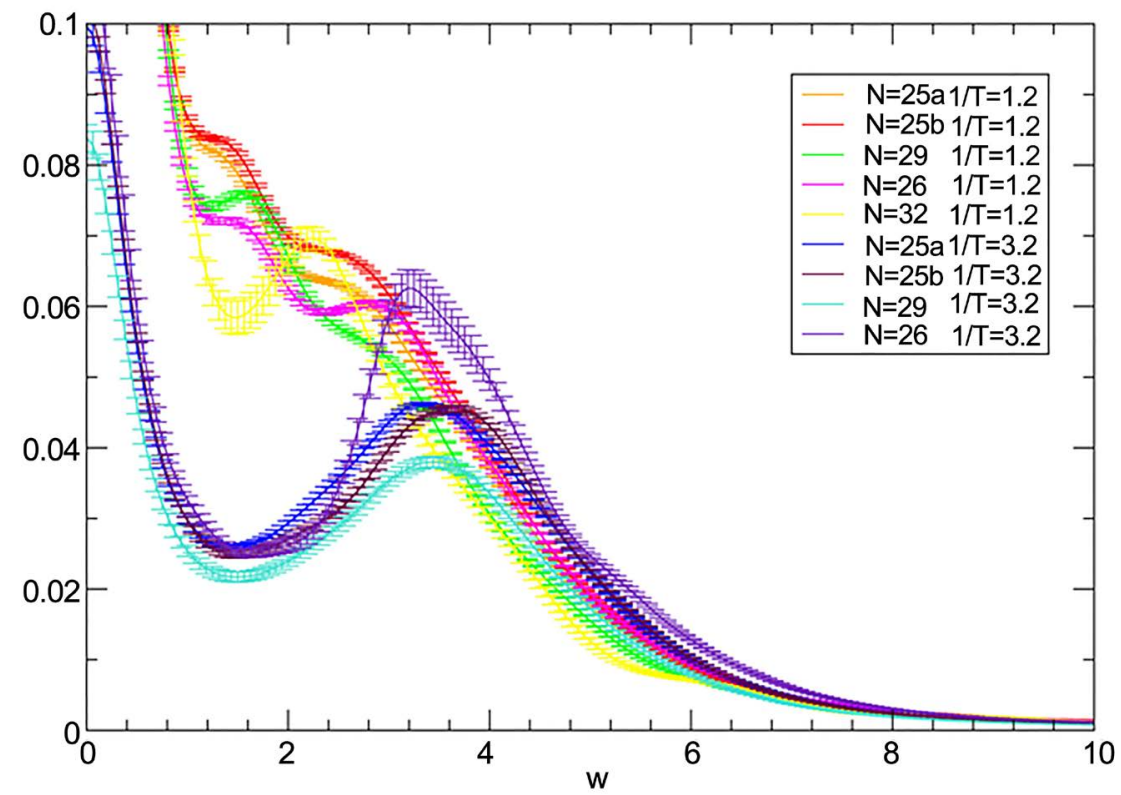

Figure 4. The dynamical correlations with $1 / T=3.2$ and 1.2 for various lattices plotted as a function of $\omega$. The error bars are the statistical errors.

$$
\begin{gathered}
S_{R, \eta}\left(\omega, T ; N_{R}, \varepsilon_{S}\right)=G_{R, \eta}\left(\omega, T ; N_{R}, \varepsilon_{S}\right) / C \\
C=\sum_{\omega_{i}=\omega_{L}}^{\omega_{U}} G_{R, \eta}\left(\omega_{i}, T ; N_{R}, \varepsilon_{S}\right) \Delta \omega \\
\omega_{i}=\omega_{L}+i \times \Delta \omega, \quad \Delta \omega=0.0625
\end{gathered}
$$

in the range $\omega_{L}<\omega<\omega_{U}$ which covers the area of the broad peak at $T=0$, so that we can easily compare our results for the different values of $T$. We employ $\omega_{L}=\omega_{c}-2 \Gamma$ and $\omega_{U}=\omega_{c}+2 \Gamma$, using the central energy of the board peak 
$\omega_{c}$ and the width $\Gamma$ at $T=0$ [29]. In Figure 5 we plot $S_{R, \eta}\left(\omega, T ; N_{R}, \varepsilon_{S}\right)$ on the $N=25 a$ lattice as a function of $\omega$ for various values of $1 / T$ between 1.12 and 10.0. We see that the broad peak gradually disappears as $1 / T$ becomes small. For example we can clearly find the broad peak at $1 / T=3.20$, while it is not easy to find the peak when $1 / T=1.44$ and there is no peak at $1 / T=1.20$.

We would like to determine a boundary of $1 / T$ below which the broad peak vanishes. It is, however, difficult to estimate such boundary temperature because the broad peak disappears gradually when $1 / T$ decreases. Therefore we introduce two kinds of $T$, which we denote $T_{s}$ and $T_{l}$. We can insist that the broad peak exists for $T \leq T_{s}$. On the other hand we admit that it is difficult to find the broad peak for $T \geq T_{l}$. In other words $T_{s}$ is a boundary by the strict condition for the broad peak, while $T_{l}$ is a boundary by the loose condition for it. On the $N=25 a$ lattice we estimate that $1 / T_{s}=2.24$ and $1 / T_{l}=1.76$.

On other lattices we can determine $T_{s}$ and $T_{l}$ in the same way. When the lattice size $N$ is odd we observe that $1 / T_{s}$ and $1 / T_{l}$ scarcely depend on $N$. In Figure 6 we show the normalized correlations $S_{R, \eta}\left(\omega, T ; N_{R}, \varepsilon_{S}\right)$ for $1 / T_{s}$ and those for $1 / T_{l}$ on the $N=25 a, 25 b$ and 29 lattices. On the even-size lattices of $N=20,26$ and 32, on the contrary, the shapes differ from each other. As a result we cannot determine a common value for $1 / T_{s}$ or $1 / T_{l}$. Our estimations are $1 / T_{s}=1.60,1 / T_{l}=1.36$ for the $N=20$ lattice, $1 / T_{s}=1.60$, $1 / T_{l}=1.20$ for the $N=26$ lattice and $1 / T_{s}=1.76,1 / T_{l}=1.44$ for the $N=32$ lattice. In Figure 7 we plot the normalized correlations for $1 / T_{s}$ and those for $1 / T_{l}$ on the $N=20,26$ and 32 lattices.

Such difference between the results on the odd-size lattices and those on the even-size lattices is ascribable to the behavior of the correlations at $T=0$ [29]. As shown in Figure 6 and Figure 7, values of $\omega_{c}$ fluctuate more on the even-

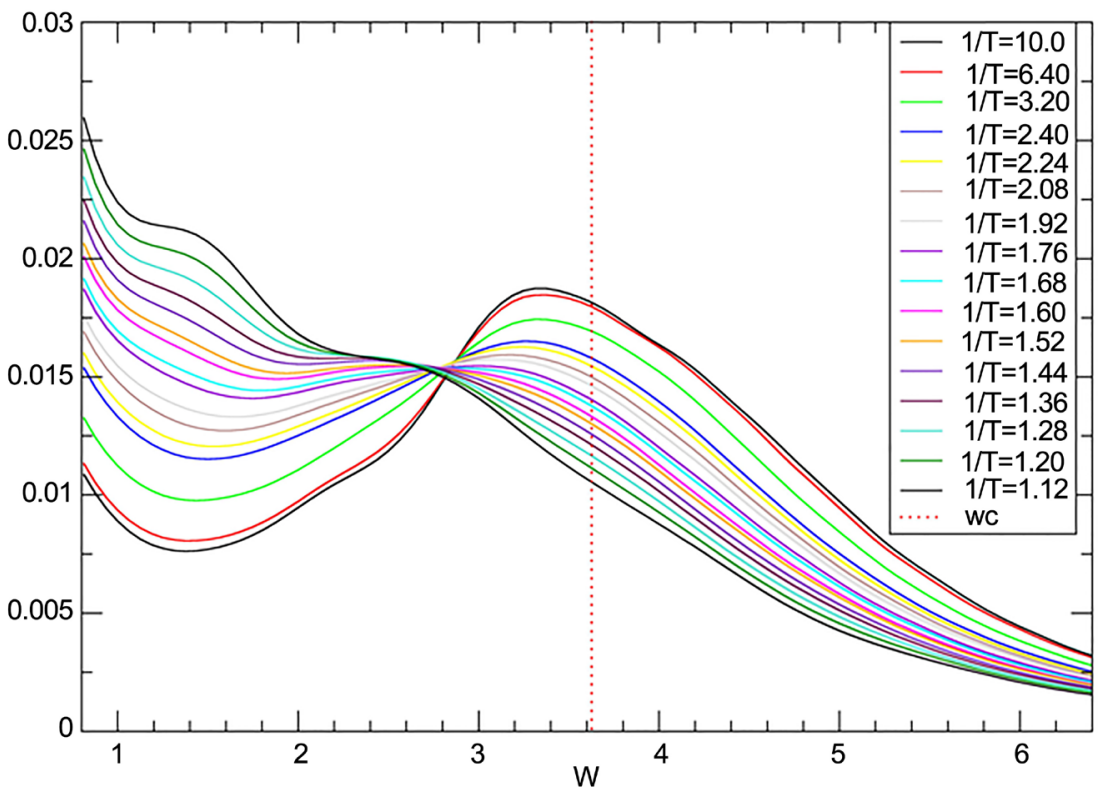

Figure 5. The normalized dynamical correlations for various values of $T$ on the $N=25 a$ lattice. The vertical red dotted line shows the value of $\omega_{c}$. 


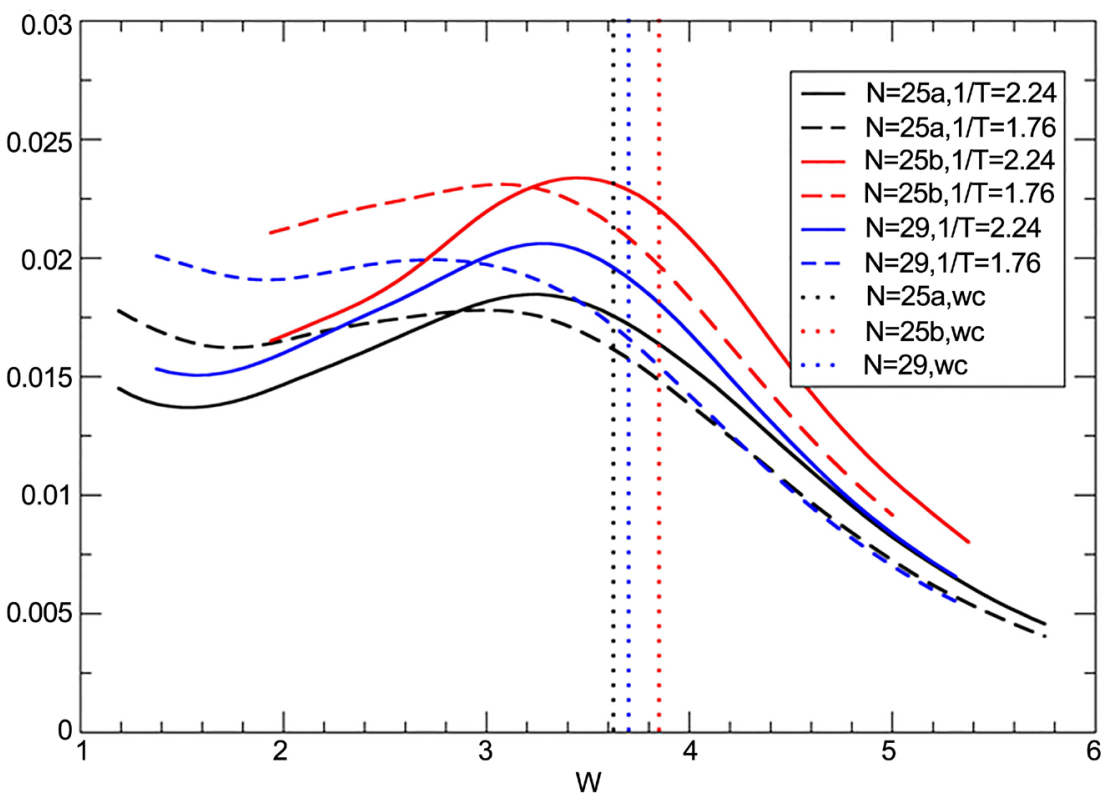

Figure 6. The dynamical correlations for $T_{s}$ and $T_{l}$ on the odd-size lattices. The vertical dotted lines show the values of $\omega_{c}$.

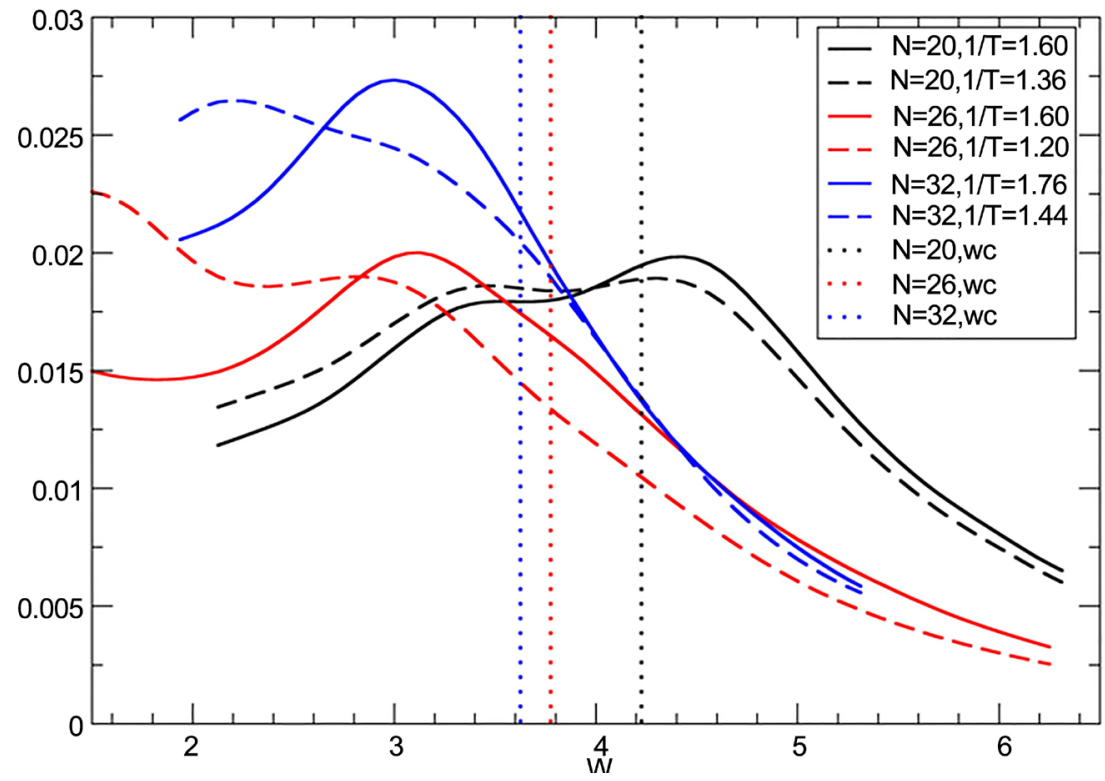

Figure 7. The dynamical correlations for $T_{s}$ and $T_{l}$ on the even-size lattices. The vertical dotted lines show the values of $\omega_{c}$.

size lattices than those on the odd-size lattices. Especially we observe that $\omega_{c}(N=20) \gg \omega_{c}(N=26,32)$. As for the height of the broad peak, it becomes large when the width $\Gamma$ is small because the normalization factor $C$ in (42) is calculated in the range between $\omega_{c}-2 \Gamma$ and $\omega_{c}+2 \Gamma$. Values of the width we calculated are $\Gamma(N=25 a)=1.41, \Gamma(N=25 b)=1.09$, and $\Gamma(N=29)=1.18$, while $\Gamma(N=20)=1.06, \Gamma(N=26)=1.24$, and $\Gamma(N=32)=0.86$.

The behavior of the dynamical correlations on the $N=20$ lattice is much 
different from those on larger lattices. It seems to be that the finite size effect is quite severe for this lattice size. Except for the $N=20$ case we see the position of the broad peak shifts to lower $\omega$ when $T>0$, for which the Higgs mode with the total spin $J>0$ should contribute in addition to the mode with $J=0$.

\section{Summary and Discussion}

In this research we have calculated, in order to find the Higgs mode, the dynamical correlations of the two spin operators in the spin-1/2 Heisenberg antiferromagnet on the square lattice at finite temperature. We have proposed an improved finite temperature Lanczos method using the stochastic state selection method for calculations on the lattices of up to 32 sites.

In the standard finite temperature Lanczos method we generate Lanczos states, calculate the eigenvalues and calculate matrix elements that are the values of the operator between two Lanczos states. In calculations of the matrix elements we have to keep the set of Lanczos states on the computer memory. Therefore the memory limits the system size for which we can calculate the matrix elements. Here we have proposed the application of the stochastic state selection method in order to weaken this limitation. This method is to select some parts of basis states stochastically and to abandon other basis states. Only by the selected basis states we calculate the inner product. After we make the statistical average, we can obtain the correct value of the inner product. By the stochastic state selection method we can drastically reduce the number of the basis states for the calculations.

In order to study the Higgs mode at finite temperature, we have calculated the dynamical correlations of the two spin operators in the spin-1/2 Heisenberg antiferromagnet on the square lattice, using the improved finite temperature Lanczos method. In calculations on the $N=20 \sim 32$ lattices we have found the broad peaks at low $T$, while there have been no peaks relevant with the Higgs mode at high $T$. Also we have found that the broad peak disappears gradually in the range $1.76<1 / T<2.24$ on the odd-size lattices, and it disappears gradually in the range $1.20<1 / T<1.76$ on the even-size lattices.

A few comments are in order. The first comment is about the parameter $M$ of the Lanczos state number. In the present calculations we used the value of $M=50$. At quite high temperature we need the larger value of $M$, because many states contribute to the correlations. In a case of $N=20$ and $1 / T=0.40$, for an example, we find that the curve of the dynamical correlations vibrates when $M=50$ whereas we find the smooth curve when $M=100$. We conclude that the vibration is unphysical and originates from the smallness of $M$.

The second comment is about $N_{s m}$, i.e. about the number of the sampling on the probability variables in the SSS method. In our calculations we have two kinds of the sampling, that are the sampling on the random state and the sampling on the SSS variables. In calculations of $G_{R, \eta}\left(\omega, T ; N_{R}, \varepsilon_{S}\right)$ in (39), we set $N_{s m}=1$. It is possible to obtain the correct values of $G_{R}(\omega, T)$ when we make the sampling on the random state by large $N_{R}$ after making the sampling on 
the probability variables $\eta$ by large $N_{s m}$ for each random state. But it is also possible to make the sampling on the probability variables $\eta$ and the sampling on the random state at the same time. This sampling means that we make one sampling on the probability variables of $\eta$, i.e. $N_{s m}=1$, for each random state $|R\rangle$. Adopting this sampling method, we can obtain the correct values of $G_{R}(\omega, T)$ if we make the sampling by large $N_{R}$. The discussion on $N_{s m}=1$ was made in the study of the SSS method [43] extensively.

The following three comments are about subjects for future study to be pursued. Since we employ the exact diagonalization approaches, the lattice size is severely limited even in the improved FTLM. Therefore it is desirable to make further study by other calculation methods, the high temperature expansions for example, which do not depend on the lattice size.

The results of this work and the previous work [29] suggest that one can find the Higgs mode in experiments of the quantum antiferromagnet on the square lattice if we measure the dynamical correlations of the two spin operators.

Another subject is about universality of the Higgs mode in the quantum spin systems. On the universality study the system on the triangle lattice is quite interesting because this system has the three kinds of the Nambu-Goldstone modes, whereas the system on the square lattice has the two kinds. It means that there must be an essential difference between both systems. Therefore it is very important to ask about what is the difference between the Higgs modes in these systems.

\section{Acknowledgements}

T. M. would like to thank Dr. Yasuko Munehisa for critical reading of the manuscript and for useful discussions.

\section{References}

[1] Higgs, P.W. (1964) Broken Symmetries, Massless Particles and Gauge Fields. Physics Letter, 12, 132-133. https://doi.org/10.1016/0031-9163(64)91136-9

[2] Aad, G., et al. (2012) Observation of a New Particle in the Search for the Standard Model Higgs Boson with the ATLAS detector at the LHC. Physics Letters B, 716, 1-29. https://doi.org/10.1016/j.physletb.2012.08.020

[3] Chatrchyan, S., et al. (2012) Observation of a New Boson at a Mass of $125 \mathrm{GeV}$ with the CMS Experiment at the LHC. Physics Letters B, 716, 30-61. https://doi.org/10.1016/j.physletb.2012.08.021

[4] Pekker, D. and Varma, C.M. (2015) Amplitude/Higgs Modes in Condensed Matter Physics. Annual Review of Condensed Matter Physics, 6, 269-297. https://doi.org/10.1146/annurev-conmatphys-031214-014350

[5] Endres, M., Fukuhara, T., Pekker, D., Cheneau, M., Schaub, P., Gross, C., Demler, E., Kuhr, S. and Bloch, I. (2012) The "Higgs" Amplitude Mode at the Two-Dimensional Super Fluid/Mott Insulator Transition. Nature, 487, 454-458. https://doi.org/10.1038/nature11255

[6] Ruegg, Ch., Normand, B., Matsumoto, M., Furrer, A., McMorrow, D. F., Kramer, K. W., Gudel, H.-U., Gvasaliya, S. N., Mutka, H. and Boehm, M. (2008) Quantum Magnets under Pressure: Controlling Elementary Excitations in TlCuCl3. Physical 
Review Letters, 100, Article ID: 205701.

https://doi.org/10.1103/physrevlett.100.205701

[7] Matsunaga, R., Hamada, Y., Makise, K., Uzawa, Y., Terai, H., Wang, Z. and Shimano, R. (2013) Higgs Amplitude Mode in the BCS Superconductors Nb1-xTixN Inducedby Terahertz Pulse Excitation. Physical Review Letters, 111, Article ID: 057002. https://doi.org/10.1103/PhysRevLett.111.057002

[8] Measson, M.-A., Gallais, Y., Cazayous, M., Clair, B., Rodiere, P., Cario, L. and Sacuto, A. (2014) Amplitude Higgs mode in the $2 \mathrm{H}-\mathrm{NbSe} 2$ superconductor. Physical Review B, 89, Article ID: 060503. https://doi.org/10.1103/PhysRevB.89.060503

[9] Bjerlin, J., Reimann, S.M. and Bruun, G.M. (2016) Few-Body Precursor of the Higgs Mode in a Fermi Gas. Physical Review Letters, 116, Article ID: 155302.

https://doi.org/10.1103/physrevlett.116.155302

[10] Grenier, B., Petit, S., Simonet, V., Canevet, E., Regnault, L-P., Raymond, S., Canals, B., Berthier, C. and Lejay, P. (2015) Longitudinal and Transverse Zeeman Ladders in the Ising-Like Chain Antiferromagnet. Physical Review Letters, 114, Article ID: 017201. https://doi.org/10.1103/PhysRevLett.114.017201

[11] Kemper, A.F., Sentef, M.A., Moritz, B., Freericks, J.K. and Devereaux, T.P. (2015) Direct Observation of Higgs Mode Oscillations in the Pump-Probe Photoemission Spectra of Electron-Phonon Mediated Superconductors. Physical Review B, 92, Article ID: 224517. https://doi.org/10.1103/physrevb.92.224517

[12] Sherman, D., Pracht, U.S., Gorshunov, B., Poran, S., Jesudasan, J., Chand, M., Raychaudhuri, P., Swanson, M., Trivedi, N., Auerbach, A., Scheffler, M., Frydman, A. and Dressel, M. (2015) The Higgs Mode in Disordered Superconductors Close to a Quantum Phase Transition. Nature Physics, 11, 188-192. https://doi.org/10.1038/nphys3227

[13] Podolsky, D., Auerbach, A. and Arovas, D. (2011) Visibility of the Amplitude (Higgs) Mode in Condensed Matter. Physical Review B, 84, Article ID: 174522. https://doi.org/10.1103/physrevb.84.174522

[14] Barlas, Y. and Varma, C.M. (2013) Amplitude or Higgs Modes in D-Wave Superconductors. Physical Review B, 87, Article ID: 054503. https://doi.org/10.1103/PhysRevB.87.054503

[15] Tsuchiya, S., Ganesh, R. and Nikuni, T. (2013) Higgs Mode in a Superfluid of Dirac Fermions. Physical Review B, 88, Article ID: 014527. https://doi.org/10.1103/physrevb.88.014527

[16] Liu, B., Zhai, H. and Zhang, S. (2016) Evolution of the Higgs Mode in a Fermion Superfluid with Tunable Interactions. Physical Review A, 93, Article ID: 033641. https://doi.org/10.1103/physreva.93.033641

[17] Nakayama, T., Danshita, I., Nikuni, T. and Tsuchiya, S. (2015) Fano Resonance through Higgs Bound States in Tunneling of Nambu-Goldstone Modes. Physical Review A, 92, Article ID: 043610. https://doi.org/10.1103/physreva.92.043610

[18] Weidinger, S.A. and Zwerger, W. (2015) Higgs Mode and Magnon Interactions in 2D Quantum Antiferromagnets from Raman Scattering. European Physical Journal $B, \mathbf{8 8}, 237$. https://doi.org/10.1140/epjb/e2015-60438-1

[19] Bruun, G.M. (2014) Long-Lived Higgs Mode in a Two-Dimensional Confined Fermi System. Physical Review A, 90, Article ID: 023621.

https://doi.org/10.1103/physreva.90.023621

[20] Matsumoto, M. (2014) Electromagnon as a Probe of Higgs (Longitudinal) Mode in Collinear and Noncollinear Magnetically Ordered States. Journal of the Physical Society of Japan, 83, Article ID: 084704. https://doi.org/10.7566/jpsj.83.084704 
[21] Xian, Y. and Merdan, M. (2014) Longitudinal Excitations in Bipartite and Hexagonal Antiferromagnetic Spin Lattices. Journal of Physics: Conference Series, 529, Article ID: 012020. https://doi.org/10.1088/1742-6596/529/1/012020

[22] Yi-Xiang, Y., Ye, J. and Liu, W.-M. (2013) Goldstone and Higgs Modes of Photons inside a Cavity. Scientific Report, 3, 3476.

[23] Gazit, S., Podolsky, D., Auerbach, A. and Arovas, D. (2013) Dynamics and Conductivity near Quantum Criticality. Physical Review B, 88, Article ID: 235108. https://doi.org/10.1103/PhysRevB.88.235108

[24] Rancon, A. and Dupuis, N. (2014) Higgs Amplitude Mode in the Vicinity of a (2+1)-Dimensional Quantum Critical Point. Physical Review B, 89, Article ID: 180501. https://doi.org/10.1103/PhysRevB.89.180501

[25] Gazit, S., Podolsky, D. and Auerbach, A. (2013) Fate of the Higgs Mode near Quantum Criticality. Physical Review Letters, 110, Article ID: 140401. https://doi.org/10.1103/physrevlett.110.140401

[26] Katan, Y.T. and Podolsky, D. (2015) Spectral Function of the Higgs Mode in 4- $\varepsilon$ Dimensions. Physical Review B, 91, Article ID: 075132. https://doi.org/10.1103/PhysRevB.91.075132

[27] Rose, F., Leonard, F. and Dupuis, B. (2015) Higgs Amplitude Mode in the Vicinity of a (2+1)-Dimensional Quantum Critical Point: A Nonperturbative Renormalization-Group Approach. Physical Review B, 91, Article ID: 224501. https://doi.org/10.1103/physrevb.91.224501

[28] Nishiyama, Y. (2015) Critical Behavior of the Higgs- and Goldstone-Mass Gaps for the Two-Dimensional S=1 XY Model. Nuclear Physics B, 897, 555-562. https://doi.org/10.1016/j.nuclphysb.2015.06.006

[29] Munehisa, T. (2015) Numerical Study of the Higgs Mode in the Heisenberg Antiferromagnet on the Square Lattice. World Journal of Condensed Matter Physics, 5, 261-274. https://doi.org/10.4236/wjcmp.2015.54027

[30] Hatano, N. and Suzuki, M. (1993) Quantum Monte Carlo Methods in Condensed Matter Physics. World Scientific, Singapore, 13-47. https://doi.org/10.1142/9789814503815_0002

[31] De Raedt, H. and von der Linden, W. (1995) The Monte Carlo Method in Condensed Matter Physics. Springer, Berlin, 249-284.

[32] Richter, J., Schulenburg, J. and Honecker, A. (2004) Quantum Magnetism. In: Schollwock, U., Richter, J., Farnell, D.J.J. and Bishop, R.F., Eds., An Introduction to Quantum Spin Systems, Springer, Berlin, 135-152.

[33] Auerbach, A. (1994) Interacting Electrons and Quantum Magnetism. Springer, Berlin.

[34] Jaklic, J. and Prelpvsek, P. (1994) Lanczos Method for the Calculation of Finite-Temperature Quantities in Correlated Systems. Physical Review B, 49, 5065 5068. https://doi.org/10.1103/PhysRevB.49.5065

[35] Long, M.W., Prelovsek, P., El Shawish, S., Karadamoglou, J. and Zotos, X. (2003) Finite-Temperature Dynamical Correlation Using the Microcanonical Ensemble and the Lanczos Algorithm. Physical Review B, 68, Article ID: 235106. https://doi.org/10.1103/physrevb.68.235106

[36] Jaklic, J. and Prelpvsek, P. (2000) Finite-Temperature Properties of Doped Antiferromagnets. Advance Physics, 49, 1-92. https://doi.org/10.1080/000187300243381

[37] Schnack, J. and Wendland, O. (2010) Properties of Highly Frustrated Magnetic Molecules Studied by the Finite-Temperature Lanczos Method. European Physical Journal B, 78, 535-541. https://doi.org/10.1140/epjb/e2010-10713-8 
[38] Hanebaum, O. and Schnack, J. (2014) Advanced Finite-Temperature Lanczos Method for Anisotropic Spin Systems. European Physical Journal B, 87, 194. https://doi.org/10.1140/epjb/e2014-50360-5

[39] Munehisa, T. (2014) An Improved Finite Temperature Lanczos Method and Its Application to the Spin-1/2Heisenberg Model on the Kagome Lattice. World Journal of Condensed Matter Physics, 4, 134-140. https://doi.org/10.4236/wjcmp.2014.43018

[40] Munehisa, T. and Munehisa, Y. (2003) A New Approach to Stochastic State Selections in Quantum Spin Systems. Journal of the Physical Society of Japan, 72, 2759-2765. https://doi.org/10.1143/JPSJ.72.2759

[41] Munehisa, T. and Munehisa, Y. (2004) The Stochastic State Selection Method for Energy Eigenvalues in the Shastry-Sutherland Model. Journal of the Physical Society of Japan, 73, 340-347. https://doi.org/10.1143/JPSJ.73.340

[42] Munehisa, T. and Munehisa, Y. (2004) Numerical Study for an Equilibrium in the Recursive Stochastic State SelectionMethod. arXiv: cond-mat, 0403626.

[43] Munehisa, T. and Munehisa, Y. (2004) A Recursive Method of the Stochastic State Selection for Quantum Spin Systems. Journal of the Physical Society of Japan, 73, 2245-2251. https://doi.org/10.1143/JPSJ.73.2245

[44] Munehisa, T. and Munehisa, Y. (2006) The Stochastic State Selection Method Combined with the Lanczos Approachto Eigenvalues in Quantum Spin Systems. Journal of Physics. Condensed Matter, 18, 2327-2335. https://doi.org/10.1088/0953-8984/18/7/018

[45] Munehisa, T. and Munehisa, Y. (2007) An Equilibrium for Frustrated Quantum Spin Systems in the Stochastic State Selection Method. Journal of Physics. Condensed Matter, 19, Article ID: 196202. https://doi.org/10.1088/0953-8984/19/19/196202

[46] Munehisa, T. and Munehisa, Y. (2009) A Constrained Stochastic State Selection Method Applied to Frustrated Quantum Spin Systems. Journal of Physics. Condensed Matter, 21, Article ID: 236008. https://doi.org/10.1088/0953-8984/21/23/236008

[47] Munehisa, T. and Munehisa, Y. (2010) Numerical Study of the Spin-1/2 Heisenberg Antiferromagnet on a 48-Site Triangularlattice Using the Stochastic State Selection Method. arXiv, 1081161.

[48] Hams, A. and De Raedt, H. (2000) Fast Algorithm for Finding the Eigenvalue Distribution of Very Large Matrices. Physical Review E, 62, 4365-4377. https://doi.org/10.1103/PhysRevE.62.4365

[49] Iitaka, T. and Ebisuzaki, T. (2004) Random Phase Vector for Calculatingthe Trace of a Large Matrix. Physical Review E, 69, Article ID: 057701. https://doi.org/10.1103/PhysRevE.69.057701 
Submit or recommend next manuscript to SCIRP and we will provide best service for you:

Accepting pre-submission inquiries through Email, Facebook, LinkedIn, Twitter, etc. A wide selection of journals (inclusive of 9 subjects, more than 200 journals)

Providing 24-hour high-quality service

User-friendly online submission system

Fair and swift peer-review system

Efficient typesetting and proofreading procedure

Display of the result of downloads and visits, as well as the number of cited articles Maximum dissemination of your research work

Submit your manuscript at: http://papersubmission.scirp.org/

Or contactwjcmp@scirp.org 